\title{
Programmable liquid optical interfaces
}

\author{
Nature Photonics spoke to Carl Brown from Nottingham Trent University about the creation of a \\ voltage-programmable liquid-oil surface that can rapidly switch and deflect light beams.
}

\begin{abstract}
What was the motivation for this work?
Previously I was working on liquid crystals and was interested in using them to make displays and other voltageconfigurable elements. When I came to Nottingham Trent University in 2003, I learnt that Glen McHale and Mike Newton were manipulating liquid droplets by electrowetting and were also interested in micro- and nanostructured superhydrophobic surfaces. We had the idea of investigating isotropic liquids and using them to make simple devices that would modulate the phase of light independent of the light's polarization. And we wanted liquid surfaces that were reconfigurable yet avoided the need for a contact line between the solid and the fluid surface.
\end{abstract}

\section{What are the current approaches for making reconfigurable optical elements? Well, actually there is a plethora of what you might call 'actuated technologies' out there: electro-acoustic and magneto optic modulators, various liquid-crystal devices based on different phases and geometries, micro-electromechanical devices and others. Each of these techniques performs well in specific applications. There are always trade-offs between size of components, speed, robustness, power consumption, stability and so on.}

Tell us about your approach.

Our approach is based on liquid dielectrophoresis, which generates forces in a dielectric medium where there are gradients in the electric field. Our structure consists of an array of striped electrodes. Every other electrode is earthed and the one between we bias with a voltage. Between these electrodes there are strong and highly non-uniform fields, not unlike those at the edges of capacitor plates. What we do is coat the electrodes with a film of oil, and this fluid tends to gather in the region between the electrodes because that is where the strongest dielectrophoretic forces are. That in turn removes fluid from the regions directly above the electrodes, and as a result you get a wrinkle on the surface of the oil film.

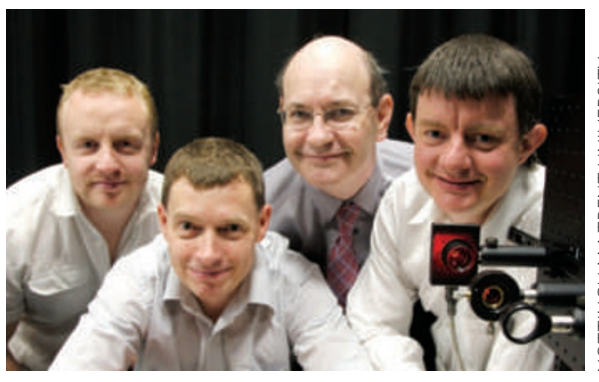

From left to right: Gary Wells, Michael Newton, Glen McHale and Carl Brown.

\section{What has been achieved with this scheme?}

We have demonstrated reconfigurable and switchable reflection and diffraction from our surface. To switch a firstorder diffracted wave's intensity we've typically used a drive voltage of $70 \mathrm{~V}_{\text {rms }}$ for a reflective device, or $190 \mathrm{~V}_{\mathrm{rms}}$ for a transmissive device. The undulation on the surface of the oil can be absolutely static and stable, and we can reproducibly reprogramme the amplitude of the wrinkle just by adjusting the potential between neighbouring electrodes. In our present work we consider oil films from 8 to $45 \mu \mathrm{m}$ in thickness and in those we can obtain peak-to-peak height of wrinkles of up to about $5 \mu \mathrm{m}$. Gary Wells, who carried out and co-designed the experimental investigations as part of his $\mathrm{PhD}$ project, came up with a neat method to pre-stretch the oil into a thin uniform film. Unlike in some other techniques, including electrowetting, we don't need to make electrical contact with any fluids in the system. We also don't have a movable contact line between our fluid and the solid surface. In electrowetting devices you need a thin dielectric layer that is capable of withstanding high electric fields. We have used a thin dielectric layer, but we don't think this is essential, because the oil film itself effectively acts as the dielectric and we use much lower electric field strengths than would cause dielectric breakdown.

How rapidly can you modify the profile? The fastest speeds we have demonstrated are for switching on the wrinkle in $35 \mu \mathrm{s}$ and relaxing (switching off) the wrinkle about $80 \mu \mathrm{s}$. In other words, if you use the periodic wrinkle as a diffraction grating, you can modulate the intensity of a first diffracted order at frequencies of about $12 \mathrm{kHz}$. A decrease in the switching speed by a factor of 10 does look feasible, but we don't know beyond that.

\section{What surface profiles can be made?}

At first we demonstrated a sinusoidal pattern down to $20 \mu \mathrm{m}$ in period. The fundamental physics doesn't change down to the micrometre scale and perhaps lower. The technical challenge is to get thinner films of oil that are uniform.

We have also demonstrated nonsinusoidal profiles, but if we introduce more gradients of the field, that costs in terms of energy. There is more work to be done there. There was a recent paper by Heikenfeld and collaborators (Nature Photon. 3, 292; 2009) showing that you can make a pixellated fluidic device with individual micrometrescale reservoirs, so we need not be limited by mass conservation. We think there is a lot more that can be done in this direction. We have shown one-dimensional profiles so far, but we think we can go to two dimensions as well.

\section{What are the applications and what are the next steps forwards? \\ We think that there are applications for optical switches and miniature projection displays, and potentially on a different scale for programmable lenticular screens to switch a display between two-dimensional and three-dimensional views. \\ We are looking at going to shorter pitches and more arbitrary profiles, and going to two dimensions. In a practical device, you may need to operate it in any orientation. In that case you need to look at using an interface between two density- matched fluids. Creating the wrinkles at liquid-liquid interfaces is going to involve some interesting and challenging physics, we think.}

\section{INTERVIEW BY DAVID PILE}

Carl Brown and his colleagues have a Letter on reconfigurable liquid surfaces on page 403 of this issue. 